\title{
Produtividade de peixes em um riacho costeiro da bacia do Leste, Rio de Janeiro, Brasil
}

\author{
Sérgio Andreas Schubart ${ }^{1} \&$ Rosana Mazzoni ${ }^{2}$
}

1. Programa de Pós-Graduação em Biologia-Ecologia, Universidade do Estado do Rio de Janeiro.

2. Laboratório de Ecologia de Peixes, Departamento de Ecologia, Universidade do Estado do Rio de Janeiro, Av. São Francisco Xavier 524, 20550-013 Rio de Janeiro, RJ. (mazzoni@uerj.br)

\begin{abstract}
Fish production in a coastal stream from Bacia do Leste, Rio de Janeiro, Brazil. Production rates (P) and biomass (B) of two stream-dwelling fish species from seven sites in the Ubatiba drainage were studied during two years with contrasting rainfall indexes. In order to test the potential correlation between P, B and the Environmental Heterogeneity Index (EHI) of each site, simple correlation analyses were applied. The analyses did not show significant differences $(\mathrm{p}<0.01)$. Spatial pattern of $\mathrm{P}$ indicated that production rates of both species are highly homogeneous in the studied sites but are low when compared with other stream-dwelling fishes from the Holartic region. Mean P and B values of Astyanax hastatus Myers, 1928 were $\mathrm{P}_{\text {rainy }}=14.0 \mathrm{~kg} \cdot \mathrm{ha}^{-1} \cdot$ year $^{-1}, \mathrm{P}_{\mathrm{dry}}=24.4 \mathrm{~kg} \cdot \mathrm{ha}^{-1} \cdot$ year $^{-1}$ and $\mathrm{B}_{\mathrm{rain}}=7.3 \mathrm{~kg} \cdot \mathrm{ha}^{-1}, \mathrm{~B}_{\mathrm{dry}}=12.2 \mathrm{~kg} \cdot \mathrm{ha}^{-1}$, with significant higher values during the dry year $(\mathrm{t}(\mathrm{B})=2.41 ; \mathrm{p}=0.03$ e $\mathrm{t}(\mathrm{P})=2.28 ; \mathrm{p}=0.04)$. In spite of presenting a tendency similar to that found for A. hastatus, mean $\mathrm{P}$ and $\mathrm{B}$ values for Geophagus brasiliensis (Quoy \& Gaimardi, 1824) did not show significant differences $(\mathrm{t}(\mathrm{B})=1.5 ; \mathrm{p}=0.16$ and $\mathrm{t}$ $(\mathrm{P})=1.75 ; \mathrm{p}=0.11$ ) between years: $\mathrm{P}_{\text {rainy }}=25.2 \mathrm{~kg} \cdot \mathrm{ha}^{-1} \cdot \mathrm{year}^{-1}, \mathrm{P}_{\text {dry }}=53.2 \mathrm{~kg} \cdot \mathrm{ha}^{-1} \cdot$ year $^{-1}$ and $\mathrm{B}_{\text {rainy }}=16.6 \mathrm{~kg} \cdot \mathrm{ha}^{-1}, \mathrm{~B}_{\mathrm{dry}}=29.7 \mathrm{~kg} \cdot \mathrm{ha}^{-1}$. Reduction of $\mathrm{P}$ in the rainy year were $57 \%$ and $47 \%$ for A. hastatus and G. brasiliensis, respectively. Mean values of $\mathrm{P} / \mathrm{B}$ decreased among larger individuals of both species.
\end{abstract}

KEYWORDS. Secondary production, turnover rates, fish, stream

RESUMO. As taxas de produção (P) e biomassa (B) de duas espécies de peixes de riacho foram estudadas em sete localidades da bacia de drenagem do rio Ubatiba, considerando-se dois ciclos anuais com índices de pluviosidade contrastantes. Com o objetivo de testar correlações potenciais entre P, B e o Índice de Heterogeneidade Ambiental (IHA) de cada localidade foram utilizadas correlações simples, que não revelaram diferenças significativas $(\mathrm{p}<0,01)$. O padrão espacial de $\mathrm{P}$ indicou que as taxas de produção de ambas as espécies foram marcadamente homogêneas nas localidades de estudo, mas foram reduzidas quando comparadas com as espécies de peixes de riacho da região Holártica. Os valores médios de P e B de Astyanax hastatus Myers, 1928 foram: $\mathrm{P}_{\text {churvo }}=14,0 \mathrm{~kg} \cdot \mathrm{ha}^{-1} \cdot \mathrm{ano}^{-1}$, $\mathrm{P}_{\text {seco }}=24,4 \mathrm{~kg} \cdot \mathrm{ha}^{-1} \cdot \mathrm{ano}^{-1} \mathrm{e} \mathrm{B}_{\text {churoso }}=7,3 \mathrm{~kg} \cdot \mathrm{ha}^{-1}, \mathrm{~B}_{\text {seco }}=12,2 \mathrm{~kg} \cdot \mathrm{ha}^{-1}$, com valores significativamente superiores durante $\mathrm{o}$ ano seco $(\mathrm{t}(\mathrm{B})=$ $2,41 ; \mathrm{p}=0,03$ e t $(\mathrm{P})=2,28 ; \mathrm{p}=0,04)$. Apesar de ter apresentado tendência semelhante à registrada para A. hastatus, os valores de $\mathrm{P} \mathrm{e}$ $\mathrm{B}$ de Geophagus brasiliensis (Quoy \& Gaimardi, 1824) não se mostraram significativamente diferentes $(\mathrm{t}(\mathrm{B})=1,5 ; \mathrm{p}=0,16$ e t $(\mathrm{P})=$ 1,$75 ; \mathrm{p}=0,11$ ) entre os anos de estudo, sendo $\mathrm{P}_{\text {churoso }}=25,2 \mathrm{~kg} \cdot \mathrm{ha}^{-1} \cdot$ ano $^{-1}, \mathrm{P}_{\text {seco }}=53,2 \mathrm{~kg} \cdot \mathrm{ha}^{-1} \cdot$ ano $^{-1}$ e $\mathrm{B}_{\text {churoso }}=16,6 \mathrm{~kg} \cdot \mathrm{ha}^{-1}, \mathrm{~B}_{\text {seco }}=29,7$ kg.ha ${ }^{-1}$. As reduções de P durante o ano de maior pluviosidade foram de $57 \%$ e $47 \%$ para A. hastatus e G. brasiliensis, respectivamente. Os valores médios da relação P/B mostraram tendência a redução em relação ao aumento de tamanho dos indivíduos de ambas as espécies.

PALAVRAS-CHAVE. Produção secundária, taxa de renovação, peixes, riacho

A despeito dos inúmeros trabalhos existentes sobre produtividade animal de regiões de clima temperado, o termo "produtividade" ainda é utilizado de forma ambígua (MAnn \& PenczaK, 1986). Thienemann (1931) apresenta uma ampla análise crítica sobre o uso do termo e define "produção" como a quantidade de matéria orgânica elaborada em uma determinada área ou comunidade biológica e "produtividade" como a taxa em que a matéria orgânica é acumulada em uma determinada unidade de tempo. Uma outra definição para o termo produção (=produtividade) considera a quantidade total de tecido elaborado por uma população durante um certo intervalo de tempo, em uma localidade definida, incluindo o que é produzido por indivíduos que não sobreviveram até o final deste intervalo de tempo (IvLEv, 1966). Este é o conceito que tem sido mais utilizado na literatura (e.g. Hopkins, 1971; Mann \& PenczaK, 1986; Mazzoni, 1999; MAZzoni et al., 2000) e será utilizado neste trabalho.

A produção de peixes tem sido objeto de diversos estudos em lagos (RYDER, 1965; DiAnA, 1983; Kelso, 1988; RANDAl et al., 1995) e riachos (Hopkins, 1971; PENCZAK,
1981; Neves \& Pardue, 1983; Waters, 1983; Whitworth \& Strange, 1983; PenczaK et al., 1985; Mann \& PenczaK, 1986; O'Hara \& Penczak, 1987; Newman \& Waters, 1989; PenCZAK, 1992) de regiões temperadas, porém são poucos os estudos realizados em outras regiões (BISHOP, 1973; Kapetsky, 1974; Watson \& Balon, 1984; Penczak \& LASSO, 1991). Para peixes tropicais brasileiros, apenas dois trabalhos estão disponíveis na literatura (i.e. AGostinHo \& PenczaK, 1995; MAZZONi \& LoBÓn-CERviÁ, 2000), apesar de que estudos dessa natureza são fundamentais para se incluir os padrões e processos que ocorrem em riachos neotropicais em um contexto mais amplo da teoria ecológica vigente, a qual, até o momento, está amplamente baseada em padrões observados em sistemas naturais do hemisfério norte.

De acordo com os dados disponíveis na literatura, as taxas de produção variam entre ambientes lênticos e lóticos, entre regiões geográficas e entre espécies. Valores de produtividade entre 50 e $750 \mathrm{~kg} / \mathrm{ha} / \mathrm{ano}$ foram registrados em riachos do Mediterrâneo (LOBÓN-CERVIÁ \& Penczak, 1984; Penczak \& Molinski, 1984); valores 
entre 20 e $300 \mathrm{~kg} / \mathrm{ha} /$ ano foram registrados em riachos tropicais (Penczak \& Lasso, 1991; Agostinho \& PenczaK, 1995; MAZZONI \& LoBÓN-CERVIÁ, 2000) e valores entre 100 e $3.272 \mathrm{~kg} / \mathrm{ha} /$ ano foram registrados em riachos da região temperada do hemisfério norte (MORTENSEN, 1977; NEVES \& PARdue, 1983; Rodríguez \& GRANAdo, 1991). Apesar da escassez de dados sobre produtividade de peixes em lagos, existem evidências de que as taxas de produção nesses ambientes são mais elevadas do que em sistemas lóticos da mesma região (RANDAL et al., 1995).

A metodologia para a obtenção dos dados necessários à aplicação dos modelos quantitativos sobre produtividade é um dos principais problemas que impõem limites a esses estudos em sistemas tropicais, pois dentre os parâmetros utilizados nos cálculos de produtividade destacam-se o tamanho da população, a idade e crescimento dos indivíduos que a compõem e suas respectivas variações no tempo. Enquanto métodos razoavelmente precisos para determinação da idade e crescimento são facilmente aplicados em espécies de peixes da região temperada (TESCH, 1971), há ainda a necessidade de se desenvolverem métodos para a análise desses parâmetros em espécies de peixes tropicais. As elevadas e constantes temperaturas que predominam nas regiões tropicais, associadas aos longos períodos reprodutivos, às múltiplas desovas e subseqüente distribuição de tamanho multimodal de larvas e juvenis dificultam a determinação da idade dos peixes. Aliado a isso tem-se o crescimento ininterrupto que caracteriza essas populações e determina a falta de um padrão claro de marcas de crescimento em suas estruturas ósseas (MAZZONI, 1999), dificultando a estimativa de crescimento dos indivíduos.

Por outro lado, a despeito do fato de que os principais métodos existentes para estimar produção de peixes (e.g. Método da Taxa Instantânea de Crescimento - Ricker, 1946 e Allen, 1949, e Método Gráfico de Allen - Allen, 1951) pressupõem o conhecimento da idade e das taxas de crescimento individuais, que são parâmetros difíceis se serem obtidos para espécies de peixes da região tropical, Hynes (1961) e Hynes \& Coleman (1968) apresentaram uma metodologia alternativa que está baseada em outras características da história de vida (i.e. tamanho, deslocamento temporal das modas de tamanho) e vem permitindo as estimativas de produção em peixes tropicais.

Neste trabalho tivemos como objetivo determinar se as taxas de produção e biomassa de duas espécies de peixes, Astyanax hastatus Myers, 1928 e Geophagus brasiliensis (Quoy \& Gaimardi, 1824), variam em diferentes localidades ao longo de uma microbacia de drenagem da bacia do Leste, RJ, e avaliar as possíveis alterações desses dois parâmetros em função de dois ciclos anuais com índices pluviométricos contrastantes.

\section{MATERIAL E MÉTODOS}

O presente trabalho foi desenvolvido na microbacia do rio Ubatiba (22 $2^{\circ} 50^{\prime}-22^{\circ} 55^{\prime} \mathrm{S}$ e $\left.42^{\circ} 43^{\prime}-42^{\circ} 50^{\prime} \mathrm{W}\right)$, uma drenagem costeira da Serra do Espraiado localizada na vertente oriental da Serra do Mar do Rio de Janeiro. É uma bacia de pequeno porte, composta por seis riachos que variam entre $1^{\mathrm{a}}$ e $2^{\mathrm{a}}$ ordem. Foram amostradas sete localidades com distância aproximada de $4 \mathrm{~km}$ entre elas (Fig. 1). Os dados utilizados para os cálculos da produtividade foram obtidos em dois ciclos de 370 dias, entre 6 de julho de 94 e 30 de julho de 95 e entre 2 de novembro de 95 e 27 de novembro de 96 , correspondendo a 13 intervalos de 30 dias cada.

O regime pluviométrico dos dois ciclos estudados foram analisados com base nos dados obtidos junto ao $6^{\circ}$ Distrito do Instituto Nacional de Meteorologia (INMET). Considerando que apenas Índices Pluviométricos (IP) superiores a $25 \mathrm{~mm}^{3} / \mathrm{h}$ influenciam o caudal dos riachos, calculamos os IP total de cada um dos dois ciclos estudados com base na quantidade de chuvas superiores a esse valor. Para isso, computamos o volume de chuva por classe de pluviosidade (14 classes com intervalo de $5 \mathrm{~mm}^{3}$ ) com base no somatório dos

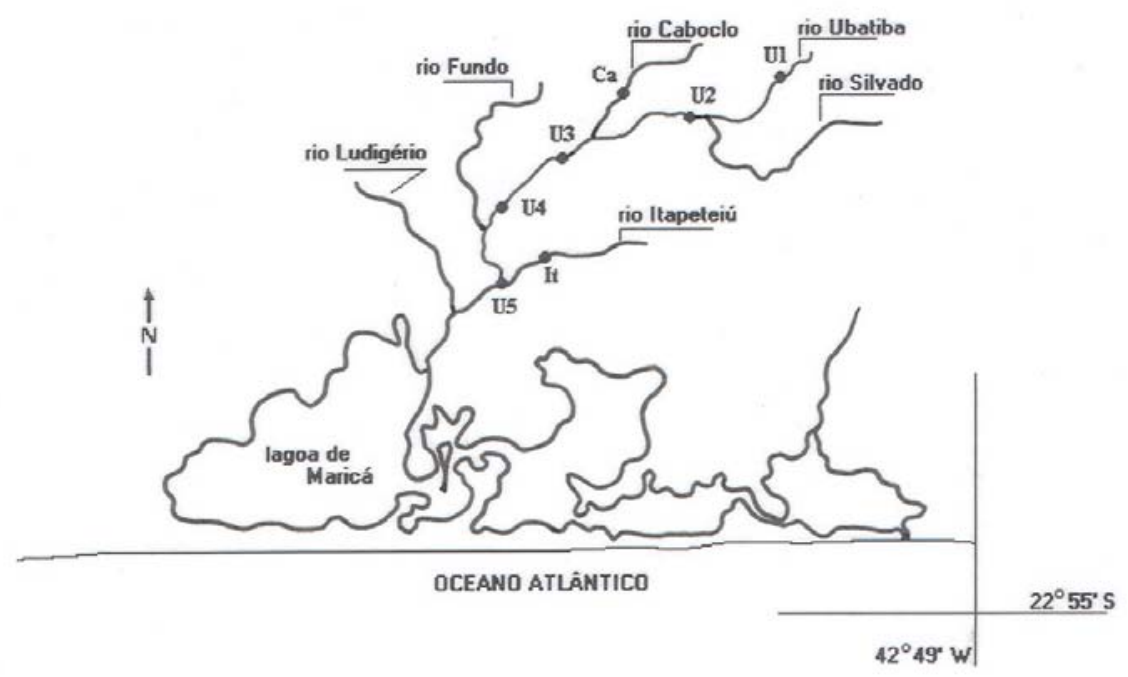

Fig. 1. Representação esquemática da bacia de drenagem do rio Ubatiba, RJ, com indicação das sete localidades estudadas. U1 a U5 são localidades na calha do rio Ubatiba e as demais são localidades nos afluentes: Ca - rio Caboclo e It - rio Itapeteiú. 
valores da pluviosidade que estavam dentro de cada intervalo de classe.

As abundâncias de Astyanax hastatus e Geophagus brasiliensis foram determinadas bimestralmente, nas sete localidades mencionadas. Para tal utilizamos a pesca elétrica $(\mathrm{CA} ; 900 \mathrm{~W}, 220 \mathrm{~V}, 1-2 \mathrm{~A}) \mathrm{e}$ o método das remoções sucessivas (ZIPPIN, 1958; Mazzoni \& Lobón-Cerviá, 2000). Para cada bimestre e localidade foram realizadas três remoções sucessivas em trechos de aproximadamente $80 \mathrm{~m}$ de extensão. Cada remoção teve duração de 20 min de maneira a se manter o esforço de coleta padronizado, conforme preconizado pelo método de ZipPIN (1958). Cada trecho de rio amostrado era fechado (nas porções inicial e final) com rede de emalhar (malha $0,5 \mathrm{~cm}$ ) de maneira a se evitar a entrada e/ ou saída dos peixes durante a amostragem. Todos os peixes coletados foram identificados, contados, medidos (comprimento padrão, $\mathrm{Cp}, \mathrm{cm}$ ) e devolvidos à água, no trecho médio da localidade amostrada; para maiores detalhes sobre a metodologia de amostragem ver MAZZONI et al., (2000). A cada ocasião e localidade de coleta foram confeccionados mapas batimétricos baseados em transectos transversais, realizados em intervalos de $5 \mathrm{~m}$, a fim de se determinar a largura, profundidade, área amostrada (Tab. I) e as características ambientais que deram origem ao Índice de Heterogeneidade Ambiental (IHA).

O IHA corresponde a uma adaptação do uso do índice de diversidade de Shannon-Wiener $\left(\mathrm{H}^{\prime}=-\Sigma \mathrm{Pi} \log \right.$ $\mathrm{Pi}$ - KREBS, 1989) para a caracterização da diversidade de cada localidade de estudo, com base na participação relativa dos componentes de três grandes variáveis ambientais, a saber: (1) SUBSTRATO do fundo do rio percentuais de matéria em decomposição, lodo (partículas $<0,03 \mathrm{~mm})$, areia $(0,03-1,0 \mathrm{~mm})$, cascalho $(1,0-5,0 \mathrm{~mm})$, seixos pequenos $(5,0-15,0 \mathrm{~mm})$, seixos grandes $(15,0$ $100,0 \mathrm{~mm})$ e rochas $(>100,0 \mathrm{~mm})$; (2) HIDROLOGIA percentuais de poças, corredeiras e rápidos; (3)
MARGEM - percentuais de vegetação ripária, vegetação aquática marginal e margem sem vegetação. Dessa forma, o IHA de cada localidade foi calculado através do somatório dos valores da equitabilidade dos diferentes componentes que compõem cada uma das três grandes variáveis ambientais mencionadas. A equitabilidade foi calculada conforme a fórmula $\mathrm{E}=\mathrm{H}^{\prime} / \mathrm{H}$ máxima, onde: $\mathrm{E}=$ Equitabilidade, H' = Diversidade de Shannon, H máxima $=\mathrm{Ln}$ de $\mathrm{S}$, e $\mathrm{S}=$ número de componentes de cada variável ambiental (PIELOU, 1974). O IHA varia de 0 a 3 e quanto maior o valor obtido, maior a heterogeneidade ambiental da localidade (Tab. II).

As densidades de A. hastatus e G. brasiliensis foram estimadas com base no método de ZIPPIN (1958), que só pode ser desenvolvido de forma iterativa, e seu modelo, baseado na função de máxima probabilidade, descreve a esperança matemática do número de indivíduos da população seguindo o modelo a seguir:

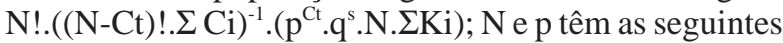
soluções: $\mathrm{N}=\mathrm{Ct} /\left(1-\mathrm{q}^{\mathrm{s}}\right)$ e q/p-s.q $/\left(1-\mathrm{q}^{\mathrm{s}}\right)=\Sigma(\mathrm{i}-1) \cdot \mathrm{Ci} / \mathrm{Ct}=\mathrm{R}$; onde $\mathrm{N}$ é o número máximo de indivíduos possíveis na unidade amostral (número que se pretende determinar); Ct é o número de indivíduos capturados; Ci é o número de indivíduos obtidos em cada remoção i (três remoções no presente trabalho); $\mathrm{p}$ é a probabilidade de que um indivíduo esteja na unidade amostral; q é o complemento de p (=1-p); K é a capturabilidade, e s é o número total de remoções em que se exerce o esforço de captura. Os cálculos do modelo de Zippin foram realizados por um programa para PC (linguagem BASIC) disponível em LOBÓN-CERVIÁ (1991).

Devido às dificuldades inerentes à determinação da idade de peixes tropicais, as taxas de produção $(\mathrm{P})$ de A. hastatus e G. brasiliensis foram estimadas pelo Método da Distribuição de Tamanho (HyNes, 1961; HynEs \& Coleman, 1968; Hamilton, 1969; Benke, 1979), pois este tem a vantagem de utilizar as coortes (modas de

Tabela I. Valores médios e desvio padrão da largura $(\mathrm{L})$, profundidade máxima $\left(\mathrm{P}_{\text {máx }}\right)$, profundidade média $\left(\mathrm{P}_{\mathrm{média}}\right)$ e área média $\left(\mathrm{A}_{\mathrm{média}}\right)$ das sete localidades amostradas no sistema fluvial do rio Ubatiba, RJ, a partir de transectos transversais realizados a intervalos de 5 m. As localidades U1 a U5 correspondem às cinco localidades estudadas no próprio rio Ubatiba e as localidades Ca e It correspondem aos seus afluentes, rios Caboclo e Itapeteiú, respectivamente.

\begin{tabular}{lcccr}
\hline Localidade & $\mathrm{L}(\mathrm{m})$ & $\mathrm{P}_{\text {máx }}(\mathrm{cm})$ & $\mathrm{P}_{\text {média }}(\mathrm{cm})$ & $\mathrm{A}_{\text {média }}(\mathrm{m})$ \\
\hline $\mathrm{U} 1$ & $1,89 \pm 0,89$ & $0,35 \pm 0,08$ & $0,14 \pm 0,09$ & $102,59 \pm 27,0$ \\
$\mathrm{U} 2$ & $1,66 \pm 0,86$ & $0,53 \pm 0,09$ & $0,24 \pm 0,11$ & $133,22 \pm 51,3$ \\
$\mathrm{U} 3$ & $1,78 \pm 0,80$ & $0,55 \pm 0,13$ & $0,26 \pm 0,13$ & $112,26 \pm 28,4$ \\
$\mathrm{U} 4$ & $2,75 \pm 1,01$ & $0,67 \pm 0,17$ & $0,24 \pm 0,15$ & $186,94 \pm 68,5$ \\
U5 & $2,48 \pm 0,95$ & $0,51 \pm 0,13$ & $0,26 \pm 0,14$ & $123,18 \pm 24,7$ \\
Ca & $1,38 \pm 0,73$ & $0,64 \pm 0,28$ & $0,25 \pm 0,19$ & $74,15 \pm 42,7$ \\
It & $1,84 \pm 0,59$ & $0,29 \pm 0,14$ & $0,11 \pm 0,06$ & $124,58 \pm 37,9$ \\
\hline
\end{tabular}

Tabela II. Índice de heterogeneidade ambiental (IHA), obtido pelo somatório dos valores de equitabilidade para Hidrologia, Substrato e Margem, das localidades estudadas no sistema fluvial do rio Ubatiba, RJ. As localidades U1 a U5 correspondem às cinco localidades estudadas no próprio rio Ubatiba e as localidades $\mathrm{Ca}$ e It correspondem aos seus afluentes, rios Caboclo e Itapeteiú, respectivamente.

\begin{tabular}{lcccccccc}
\hline & $\mathrm{U} 1$ & $\mathrm{U} 2$ & $\mathrm{U} 3$ & $\mathrm{U} 4$ & $\mathrm{U} 5$ & $\mathrm{Ca}$ \\
\hline Hidrologia & 0,939 & 0,878 & 0,716 & 0,467 & 0,659 & 0,732 & 0,634 \\
Substrato & 0,786 & 0,644 & 0,790 & 0,814 & 0,721 & 0,910 & 0,735 \\
Margem & 0,469 & 0,469 & 0,000 & 0,323 & 0,637 & 0,000 & 1,000 \\
\hline Somatório (IHA) & 2,194 & 1,991 & 1,506 & 1,604 & 2,017 & 1,642 \\
\hline
\end{tabular}


comprimento) sem a necessidade do conhecimento da idade dos peixes. $\mathrm{O}$ método mencionado tem como base as variações temporais das densidades (ind.ha ${ }^{-1}$ ) dos indivíduos que permanecem durante um intervalo de tempo em um determinado intervalo de tamanho (classe de comprimento, $\mathrm{cm}$ ). Os intervalos de tamanho foram estabelecidos com base no comprimento máximo de cada espécie (intervalos de $5 \mathrm{~mm}$ e $10 \mathrm{~mm}$ para A. hastatus e $G$. brasiliensis, respectivamente). O somatório dos deslocamentos das classes de comprimento em cada intervalo de tempo, que representam as variações nos números de indivíduos de cada intervalo de tamanho, equivale ao crescimento dos indivíduos nesse intervalo, durante o tempo entre as amostragens (60 dias no presente trabalho), subtraído o número de indivíduos que morreram ou que, alternativamente, foram incorporados à população por imigração ou recrutamento. Essas variações representam a produção do conjunto de indivíduos desse intervalo de tamanho ao longo do tempo entre as amostras. Como consequiência, o somatório das variações observadas em todos os intervalos de tamanho entre todos os intervalos de tempo ( 7 intervalos de 60 dias para cada ano estudado no presente trabalho) representa a produção total da população.

Outro fator requerido para o cálculo da produção é o peso médio anual em cada intervalo de comprimento da população. Ele é expresso como: w.k $=\left(\Sigma\left(D_{i+1}{ }^{-}\right.\right.$ $\left.\left.\mathrm{D}_{\mathrm{i}}\right) .\left(\mathrm{w}_{\mathrm{i}} \mathrm{k}+\mathrm{w}_{\mathrm{i}}+1 \mathrm{k}\right) / 2\right) .(\mathrm{Da}-\mathrm{D} 1)^{-1}$, onde $\mathrm{w}_{\mathrm{i}} \mathrm{k}$ é o peso médio dos indivíduos dentro do intervalo de tamanho $\mathrm{k}$ na data $\mathrm{i}$; Da-D1 corresponde ao total de dias que envolveu o estudo.

O cálculo da produção anual (Ps - f) pelo Método de Distribuição de Tamanhos, usando a abundância anual média e o peso anual médio para cada intervalo de comprimento, é estimado por: Ps- $\mathrm{f}=0,5 \mathrm{C} .\left(\mathrm{w}_{\mathrm{i}}\left(\mathrm{N}_{\mathrm{i}}-\right.\right.$ $\left.\mathrm{N}_{\mathrm{i}+1} \cdot 2\right)+\sum \mathrm{w} \cdot \mathrm{k}(\mathrm{N} \cdot \mathrm{k}-1-\mathrm{N} \cdot \mathrm{k}+1)+\mathrm{w} . \mathrm{c}(\mathrm{N} \cdot \mathrm{c}-1-\mathrm{N} . \mathrm{c}) \cdot(1 / \mathrm{CPI})^{-1}$, onde $\mathrm{N}$ representa a abundância anual média em cada intervalo de comprimento $\mathrm{k}$ na amostragem $\mathrm{i} ; \mathrm{w}_{\mathrm{i}}$ é o peso médio dos indivíduos; CPI é a idade máxima média da coorte em anos.

Considerando que durante as amostragens o peso dos exemplares coletados não foi registrado, devido a questões práticas de manipulação de indivíduos vivos, os pesos médios foram obtidos a partir dos coeficientes "a" e "b" das relações peso-comprimento, obtida para cada espécie a partir de indivíduos coletados mensalmente para estudos paralelos sobre a biologia das mesmas. Para A. hastatus temos: $\mathrm{a}=0,02652(\mathrm{Lna}=-3,63)$ e $\mathrm{b}=2,98$, com $\mathrm{r}=0,89$, para 227 indivíduos analisados. Para $G$. brasiliensis estes valores são: $\mathrm{a}=0,04736(\mathrm{Lna}=-3,05) \mathrm{e}$ $\mathrm{b}=2,89$, com $\mathrm{r}=0,97$, para 286 indivíduos analisados. Desse modo, as estimativas de produção foram feitas com base nas distribuições de freqüência de comprimento de cada uma das duas espécies, por localidade e período amostral.

De posse dos valores estimados da produção e biomassa (B), foram obtidas as taxas de renovação da biomassa (P/B) de cada espécie. Foram calculados os coeficientes de correlação simples entre os valores do IHA, Pe B (ZAR, 1999). Comparamos, ainda, a taxa de renovação média da biomassa de cada espécie, com seu respectivo comprimento (Cp) médio, em cada ano de estudo.

Para os conjuntos de dados de produção, biomassa e taxa de renovação foram aplicados testes de normalidade de Kolmogorov-Smirnov (ZAR, 1999) com correção de Lilliefors (ARANGO, 2001). Para as distribuições normais e não normais aplicamos, de acordo com o caso, os testes de variância T ou ANOVA e Kruskal-Wallis, respectivamente.

\section{RESULTADOS E DISCUSSÃO}

As localidades amostradas neste estudo foram selecionadas a fim de identificarmos possíveis relações de causalidade entre as taxas de produção (P) e biomassa (B) e a gama de condições ambientais que prevalece ao longo da bacia de drenagem do rio Ubatiba. Porém, as correlações entre P, B e o IHA não foram significativas $(\mathrm{p}<0,01)$. Diversos relatos disponíveis na literatura indicam a existência de correlação positiva entre $\mathrm{P}$ e distintas variáveis ambientais (NEWMAN \& WATERS, 1989; Agostinho \& PenczaK, 1995; Formigo \& PenczaK, 1999; Екмекс̧г, 2002). No presente trabalho, as variações espaciais das variáveis ambientais foram tratadas sob a forma do IHA, que é um índice que informa sobre a diversidade das variáveis ambientais de cada localidade. Assim sendo, apesar de não termos analisado a relação direta das variáveis ambientais com os parâmetros $\mathrm{P}$ e $\mathrm{B}$, podemos sugerir que nossos resultados contrastam com os demais disponíveis na literatura, que afirmam sobre a relação de causalidade entre os parâmetros $\mathrm{P}$ e $\mathrm{B}$ e as variáveis ambientais, destacando-se a disponibilidade de abrigo, profundidade da coluna d'água, densidade de macrófitas e condutividade da água. Vale ressaltar que todos os autores mencionados trabalharam em ambientes de clima subtropical e/ou temperado, fato que os diferencia da área de estudo do presente trabalho.

Com base na análise da pluviosidade dos dois períodos de estudo, constatamos que o período novembro de 95 -novembro de 96 foi mais chuvoso $\left(1.139,9 \mathrm{~mm}^{3}>\right.$ $25 \mathrm{~mm}^{3} / \mathrm{h}$ ) se comparado com o período julho de 94 -julho de $95\left(760,7 \mathrm{~mm}^{3}>25 \mathrm{~mm}^{3} / \mathrm{h}\right)$ (Fig. 2).

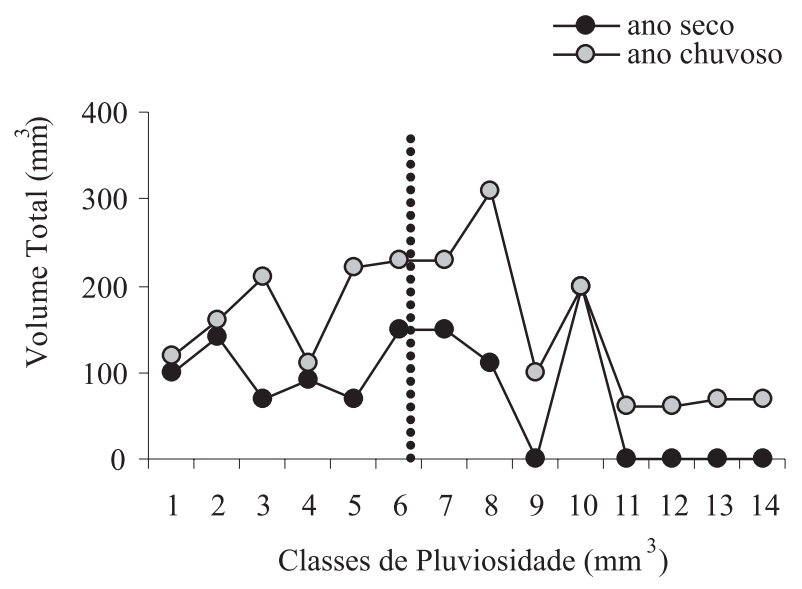

Fig. 2. Volume total de chuvas por classe de pluviosidade para os dois períodos de estudo (julho/1994 a julho/1995 e novembro/ 1995 a novembro/1996). A linha pontilhada separa o volume de chuvas que afeta o caudal do rio $\left(>25 \mathrm{~mm}^{3}\right)$ do volume de chuvas que não afeta o caudal do rio $\left(<25 \mathrm{~mm}^{3}\right)$. Classes em $\mathrm{mm}^{3}: 1=0 \mathrm{a}$ $5 ; 2=5$ a $10 ; 3=10$ a $15 ; 4=15$ a $20 ; 5=20$ a $25 ; 6=25$ a $30 ; 7$ $=30$ a $35 ; 8=35$ a $40 ; 9=40$ a $45 ; 10=45$ a $50 ; 11=50$ a $55 ; 12$ $=55$ a $60 ; 13=60$ a $65 ; 14=65$ a 70 . 
As médias anuais de biomassa $(\mathrm{B})$ e produção $(\mathrm{P})$ foram mais elevadas no período seco para $A$. hastatus ( $\mathrm{t}$ $(\mathrm{B})=2,41 ; \mathrm{p}=0,03$ e t $(\mathrm{P})=2,28 ; \mathrm{p}=0,04)$. Para $G$. brasiliensis não foram observadas diferenças significativas $(\mathrm{t}(\mathrm{B})=1,5 ; \mathrm{p}=0,16$ e $\mathrm{t}(\mathrm{P})=1,75 ; \mathrm{p}=0,11)$, porém houve tendência semelhante à registrada para $A$. hastatus (Tab. III). Dudgeon (1999), comparando a produção de uma comunidade de insetos aquáticos entre dois anos com contrastes pluviométricos, observou que as espécies que utilizavam as margens como microhábitat eram suscetíveis ao arraste durante as chuvas e apresentaram marcada redução da produtividade durante o ano chuvoso, enquanto as espécies que habitavam o trecho médio do rio, onde a velocidade da água era mais alta, foram mais abundantes e mais produtivas no ano chuvoso. Entre as espécies do presente estudo, $A$. hastatus é tipicamente da coluna d'água, explorando essencialmente as áreas de corredeira (MAzzoni et al., 2004), enquanto G. brasiliensis tem hábito bentônico e se distribui principalmente na vegetação próxima à margem (MAZZONi \& Iglesias-Rios, 2002); ambas as espécies foram mais produtivas durante o ano seco; porém, A. hastatus apresentou uma redução de $57 \%$ no período chuvoso, enquanto G. brasiliensis apresentou redução de $47 \%$. Essa observação parece refletir a influência das variações do caudal do rio sobre a produção dos organismos aquáticos mas não corrobora as observações de DUDGEON (1999). Não existem dados na literatura que permitam avaliar se o padrão observado é ou não recorrente em comunidades de peixes.

A variação espacial dos valores médios de $\mathrm{P}$ (Tab. III) indica que a produtividade de ambas as espécies varia significativamente ao longo do gradiente longitudinal da bacia de drenagem do rio Ubatiba $\left(\mathrm{F}_{\text {Astyanax }}=70,25 ; \mathrm{p}<0,001\right.$ e $\left.\mathrm{F}_{\text {Geophagus }}=24,16 ; \mathrm{p}<0,001\right)$, mas seus valores médios são bem reduzidos em relação aos valores registrados para as espécies de riachos de regiões de clima temperado (e.g., Hopkins, 1971; NeVEs \& PARDUE, 1983; LobÓN-CERVIÁ \& PenczaK, 1984; PenczaK \& Molinski, 1984; Mahon \& Balon, 1985; Rodríguez \& Granado, 1991; Cabral \& Marques, 1999, Formigo \& PenczaK, 1999). A estrutura geológica, com formações pré-cambrianas e corredeiras basálticas (PICCIRILlo et al., 1988), caracteriza os riachos neotropicais e determina ambientes pobres em nutrientes, livres de macrófitas e sujeitos a freqüentes chuvas torrenciais que agem na "lavagem" do ambiente. Tem-se ainda que a disponibilidade de alimento autóctone tornase limitada em função de seu carreamento rio abaixo (Margalef, 1983; Watson \& Balon, 1984; LoweMcConnell, 1987; PenczaK \& LAsso, 1991; MAZzoni \& LOBÓN-CERví́ 2000). As variações temporais do caudal da bacia de drenagem do rio Ubatiba são bastante elevadas durante os períodos de chuva, podendo sofrer variações de até $5 \mathrm{~m}$ na coluna d'água (MAZZONI \& LoBÓNCERviá, 2000), o que pode explicar as baixas taxas de produção para o ano chuvoso e, de maneira geral, as baixas taxas de produção do sistema como um todo.

Os valores médios da taxa de renovação da biomassa $(\mathrm{P} / \mathrm{B})$ foram significativamente diferentes entre as espécies ( $\mathrm{t}=-2,84 ; \mathrm{p}<0,008)$, sendo que $A$. hastatus, a espécie de menor porte $\left(\mathrm{Cp}_{\text {médio }}=3,15\right)$, apresenta maior $\mathrm{P} / \mathrm{B}$; além disso, para uma mesma espécie, o P/B mostrou tendência a redução durante o ano chuvoso (Fig. 3).

$\mathrm{P} / \mathrm{B} \square \mathrm{Cp}$

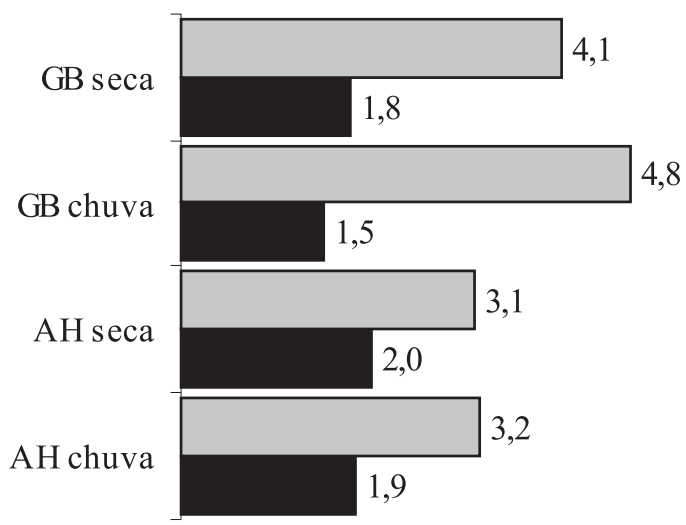

Fig. 3. Valores médios da relação produção e biomassa $(P / B)$ e comprimento padrão ( $\mathrm{Cp}$ ) para Geophagus brasiliensis nos anos seco (GB seco) e chuvoso (GB chuva) e para Astyanax hastatus nos anos seco (AH seca) e chuvoso (AH chuva).

Tabela III. Biomassa anual média B (kg.ha $\left.{ }^{-1}\right)$ e taxa de produção P (kg.ha ${ }^{-1}$.ano ${ }^{-1}$ ) para Astyanax hastatus (AH) e Geophagus brasiliensis $(G B)$ nas sete localidades amostradas no sistema fluvial do rio Ubatiba, RJ, para os ciclos seco (1994/1995) e chuvoso (1995/1996). As localidades U1 a U5 correspondem às cinco localidades estudadas no próprio rio Ubatiba e as localidades Ca e It correspondem aos seus afluentes, rios Caboclo e Itapeteiú, respectivamente.

\begin{tabular}{|c|c|c|c|c|c|c|c|c|c|c|c|c|}
\hline \multirow{3}{*}{$\frac{\text { Espécie }}{\text { Período }}$} & \multicolumn{4}{|c|}{$A H$} & \multicolumn{4}{|c|}{$G B$} & \multicolumn{4}{|c|}{ Total } \\
\hline & \multicolumn{2}{|c|}{ seco } & \multicolumn{2}{|c|}{ chuvoso } & \multicolumn{2}{|c|}{ seco } & \multicolumn{2}{|c|}{ chuvoso } & \multicolumn{2}{|c|}{ seco } & \multicolumn{2}{|c|}{ chuvoso } \\
\hline & B & $\mathrm{P}$ & B & $\mathrm{P}$ & B & $\mathrm{P}$ & B & $\mathrm{P}$ & B & $\mathrm{P}$ & B & $\mathrm{P}$ \\
\hline U1 & 9,3 & 18,9 & 4,5 & 6,9 & 35,4 & 52,3 & 9,8 & 13,7 & 44,7 & 71,2 & 14,2 & 20,6 \\
\hline $\mathrm{U} 2$ & 11,8 & 22,4 & 7,7 & 13,0 & 67,1 & 127,8 & 26,2 & 46,5 & 78,9 & 150,2 & 33,9 & 59,5 \\
\hline U3 & 9,1 & 15,6 & 6,7 & 12,5 & 36,4 & 53,1 & 34,6 & 50,0 & 45,5 & 68,7 & 41,2 & 62,5 \\
\hline U4 & 13,9 & 28,1 & 4,6 & 7,4 & 19,4 & 30,7 & 4,0 & 6,9 & 33,3 & 58,8 & 8,7 & 14,3 \\
\hline U5 & 10,3 & 23,9 & 8,5 & 17,6 & 27,4 & 62,3 & 24,0 & 33,8 & 37,7 & 86,2 & 32,5 & 51,5 \\
\hline $\mathrm{Ca}$ & 8,8 & 17,9 & 6,0 & 11,8 & 2,8 & 3,4 & 5,8 & 8,4 & 11,5 & 21,3 & 11,8 & 20,2 \\
\hline It & 20,5 & 44,0 & 13,1 & 28,6 & 19,6 & 42,8 & 11,6 & 17,4 & 40,1 & 86,8 & 24,8 & 45,9 \\
\hline Média & 12,0 & 24,4 & 7,3 & 14,0 & 29,7 & 53,2 & 16,6 & 25,2 & 41,7 & 77,6 & 23,9 & 39,2 \\
\hline Total & 83,7 & 170,8 & 51,1 & 97,7 & 208,1 & 372,3 & 116,0 & 176,8 & 291,7 & 543,1 & 167,2 & 274,5 \\
\hline
\end{tabular}


De acordo com MARGALEF (1983), a P/B em peixes varia de 0,5 a 5,0 . No entanto, valores de $\mathrm{P} / \mathrm{B}$ variando entre 0,06 e 0,93 foram registrados para diversas espécies de riachos tropicais por PENCZAK \& LASSO (1991), LOBÓNCerviá et al. (1993), Agostinho \& Penczak (1995) e Mazzoni \& Lobón-Cerviá (2000), o que coloca nossos resultados acima dos limites registrados para ambientes tropicais mas muito abaixo dos valores registrados para peixes de tamanhos semelhantes de regiões de clima temperado e mediterrâneo, que têm $\mathrm{P} / \mathrm{B}$ variando entre 3,0 e 5,0 (e.g. Mann \& PenczaK, 1986, Cabral \& Marques, 1999). Assim sendo, os baixos valores de $\mathrm{P} / \mathrm{B}$ observados neste e em outros estudos na região neotropical sugerem que essas espécies crescem menos e por menor tempo quando comparadas às espécies da região holártica. Esses resultados sugerem, ainda, que os indivíduos das espécies estudadas apresentam crescimento muito rápido em curto espaço de tempo. Um dos fatores que pode determinar esse padrão é a reprodução precoce, que, quando associada a períodos prolongados de reprodução, compensam as baixas taxas de renovação individual, com altas taxas de renovação da população. Reprodução precoce e período reprodutivo prolongado são características recorrentes entre as espécies de peixes da bacia de drenagem do rio Ubatiba (e.g. Menezes \& Caramaschi, 1994; Mazzoni \& Petito, 1999; Mazzoni et al., 2002; Mazzoni \& Iglesias-Rios, 2002). Ainda nesse contexto, os valores de $\mathrm{P} / \mathrm{B}$ registrados indicam relação inversa com o tamanho dos indivíduos, fato que está de acordo com as premissas termodinâmicas que prevêem redução da energia convertida por unidade de biomassa (Matsuno, 1978; 1984).

Agradecimentos. À equipe do Laboratório de Ecologia de Peixes, UERJ, pelo auxílio no trabalho de campo. À Érica Pellegrini Caramaschi e Ricardo Iglesias-Rios pelas sugestões no manuscrito. Á FAPERJ (Processo n ${ }^{\circ}$. E-26/171.231/98-APQ1) e CNPq (Processos n ${ }^{\circ}$. 521597/95-Proj. Integrado e 302628/2002 9-Produtividade para R.M.), pelo apoio financeiro. Este trabalho é parte da dissertação de S.A.S., (Bolsa CNPq Processo n. 130863/ 2001).

\section{REFERÊNCIAS BIBLIOGRÁFICAS}

Agostinho, A. A. \& Penczak, T. 1995. Populations and production of fish in two small tributaries of the Paraná river, Paraná, Brazil. Hydrobiology 312:153-166.

Allen, K. R. 1949. Some aspects of the production and cropping of freshwaters. Transactions Royal Society of New Zeland 77(5):222-228

1951. The Horokiwi stream. New Zeland Marine Department, Fisheries Bulletin. 238p.

Arango, H. G. 2001. Bioestatística teórica e computacional Rio de Janeiro, Guanabara Koogan. 235p.

Benke, A. C. 1979. A modification of the Hynes method for estimating secondary production with particular significance for multivoltine populations. Limnological Oceanography 24:168-171

Bishop, J. E. 1973. Limnology of a Malaian river, Sungai Gombak. Hague, W. Junk. 485p.

Cabral, J. A. \& Marques, J. C. 1999. Life history, population dynamics and production of eastern mosquitofish, Gambusia holbrooki (Pisces, Poeciliidae), in rice fields of the lowe Mondego River Valley, Western Portugal. Acta Oecologica 20 (6):607-620

Diana, J. S. 1983. Growth, maturation, and production of northern pike in three Michigan lakes. Transactions of the American Fisheries Society 112:38-46.
Dudgeon, D. 1999. Patterns of variation in secondary production in a tropical stream. Archives fur Hydrobiology 144(3): 271-281.

Екмекс̧г, F. G. 2002. The effects of high salinity on the production of Capoeta tinca in a naturally contamined river. Turkey Journal of Zoology 26:265-270.

Formigo, N. \& Penczak, T. 1999. Fish stocks of the Âncora River, northern Portugal: production. Fisheries Research 40:91-96.

Hamilton, A. L. 1969. On estimating annual production. Limnology and Oceanography 14:771-782.

Hopkins, C. L. 1971. Production of fish in two small streams in the North Island of New Zealand. Marine and Freshwater Research 5(2):280-290.

Hynes, H. B. 1961. The invertebrate fauna of a Welsh mountain stream. Archives fur Hydrobiologie 57:344-388.

Hynes, H. B. \& Coleman, M. J. 1968. A simple method of assessing the annual production of stream benthos. Limnological Oceanography 13:569-575.

IvLEv, V. S. 1966. The biological productivity of waters. Journal of Fisheries and Research Board of Canada 23(11):17271759.

KAPETSKy, J. M. 1974. The Kafue River floodplain: an example of preimpoundment potential for fish production. In: BALON, E. K. \& Coche, A. G. eds. Lake Kariba: a man-made tropical ecosystem in Central Africa. Hague, W. Junk. p.497-523.

Kelso, J. R. M. 1988. Fish community structure, biomass, and production in the Turkey Lakes watershed, Ontario. Canadian Journal of Fisheries and Aquatic Science 45:115-120.

KreBs, C. J. 1989. Ecological methodology. British Columbia, Harper Collins Publishers. $654 \mathrm{p}$.

Lobón-Cerviá, J. 1991. Dinamica de poblaciones de peces en rios: pesca eléctrica y métodos de capturas sucesivas en la estima de abundancias. Madrid, Graficas MARCAR, S.A. 156 p.

Lobón-Cerviá, J. \& Penczak, T. 1984. Fish production in the Jarama River, Central Spain. Holartic Ecology 7:128-137.

Lobón-Cerviá, J., Utrilla, C. G., Querol, E. \& Puig, M. A. 1993. Population ecology of pike-cichlid, Crenicichla lepidota, in two streams of the Brazilian Pampa subject to a severe drought. Journal of Fish Biology 43:537-557.

Lowe-McConnell, R. 1987. Ecological studies in tropical fish communities. Cambridge, Cambridge University. 382 p.

Mahon, R. \& BALON, E. K. 1985. Fish production in warmerwater streams in Poland and Ontario. Canadian Journal of Fisheries and Aquatic Science 42:1211-1215.

ManN, R. H. K. \& PenczaK, T. 1986. Fish production in rivers: a review. Polschie Archives of Hydrobiology 33:233-247.

Margalef, R. 1983. Limnología. Barcelona, Ediciones Omega. $1010 \mathrm{p}$

Matsuno, K. 1978. Evolution of dissipative system: a theoretical basis of Margalef's principle of ecosystem. Journal of Theoretical Biology 70:23-31.

1984. In beyond neo-darwinism: an introduction to the new evolutionary paradigm. Florida, Academic Press. $83 \mathrm{p}$.

Mazzoni, R. 1999. Produção de peixes em riachos: princípios, métodos e perspectivas. In: Caramaschi, E. P.; Mazzoni, R. \& Peres-Neto, P. R. eds. Ecologia de peixes de riacho. Série Oecologia Brasiliensis, vol. VI. PPGE/UFRJ/Rio de Janeiro, Computer \& Publish Editoração Ltda. p. 237-260.

Mazzoni, R. \& Iglesias-Rios, R. 2002. Environmentally related life history variations in Geophagus brasiliensis. Journal of Fish Biology 61:1606-1618.

Mazzoni, R. \& Lobón-Cerviá, J. 2000. Longitudinal structure, density and production rates of a neotropical stream fish assemblage: the river Ubatiba to the Serra do Mar, southeast Brazil. Ecography 23:588-602.

Mazzoni, R. \& Petito, J. 1999. Reproductive Biology of a Tetragonopterinae (Osteichyes, Characidae) of the Ubatiba Fluvial System, Maricá - RJ. Brazilian Archives of Biology and Technology 42(4):455-461.

Mazzoni, R.; Fenerich-Verani, N. \& Caramaschi, E. P. 2000. Electrofishing as a sampling technique for coastal stream fish populations and communities in the southeast of Brazil. Revista Brasileira de Biologia 60(2):205-216. 2002. Reproductive biology of a Characidiinae (Osteichthyes, 
Characidae) from the Ubatiba River, Maricá - RJ. Brazilian Journal of Biology 62(3):487-494.

Mazzoni, R.; Schubart, S. A. \& Iglesias-Rios, R. 2004. Movement Patterns of Astyanax janeiroensis along a small stream in Southeast Brazil. Ecology of Freshwater Fish 13:231-234.

Menezes, M. S. \& Caramaschi, E. P. 1994. Características reprodutivas de Hypostomus grupo $H$. punctatus no rio Ubatiba, Maricá, RJ (Oeteichthyes, Siluriformes). Revista Brasileira de Biologia 54(3):503-513.

Mortensen, E. 1977. Fish production in small Danish streams Folia Limnologica Scandinavica 17:21-26.

Neves, R. J. \& Pardue, G. B. 1983. Abundance and production of fishes in a small Appalachian stream. Transactions of the American Fisheries Society 112:21-26.

Newman, R. M. \& Waters, T. F. 1989. Differences in brown trout (Salmo trutta) production among contiguous sections of an entire stream. Canadian Journal of Fisheries and Aquatic Sciences 46:203-213.

O'hara, K. \& Penczak, T. 1987. Production of the three-spined stickleback, Gasterosteus aculeatus L., in the River Weaver, England. Freshwater Biology 18:353-360.

PenCZAK, T. 1981. Ecological fish production in two small Lowland rivers in Poland. Oecologia 48:107-111.

. 1992. Fish production in the Warta river, Poland: a preimpoundment study. Hydrobiologia 237:117-129.

PenczaK, T. \& Lasso, C. 1991. Problems of estimating population parameters and production of fish in a tropical rain forest stream, north Venezuela. Hydrobiologia 215:121-133.

Penczak, T. \& Molinski, M. 1984. Fish production in the Oued Sebaou, a seasonal river in north Algeria. Journal of Fish Biology 25:723-732.

PenczaK, T.; Jankov, J. \& Zalewski, M. 1985. Fish production in the Mesta river, Rila Mountain, Samokov, Bulgaria. Fisheries Research 3:201-221.

Piccirillo, E. M.; Melfi, A. J.; Comin-Chiaramonti, B.; Bellieni, G.; ERnesto, M.; Marques, L. S.; NARdi, J. R.; Pacca, E. G.; RoisenberG, A. \& Estolfa, D. 1988. Continental flood volcanism from the
Paraná basin. In: MacDougali, E. D. ed. Continental floods basalts. Dordrecht, Kluwer Academic Publisher. p.195-238.

Pielou, E. C. 1974. Population and community ecology. New York, Gordon \& Breach, Science Publishers, Inc. 424p.

Randal, R. G., Kelso, J. R. M. \& Minns, C. K. 1995. Fish production in freshwaters: Are rivers more productives than lakes? Canadian Journal of Fisheries and Aquatic Science 52:631-643.

Ricker, W. E. 1946. Production and utilization of fish populations. Ecological Monographs 16:373-391.

Rodríguez, R. A. \& Granado, L. C. 1991. Fish production in a stream with Mediterranean regimen (SW Spain). Ecological International Bulletin 19:65-78.

Ryder, R. A. 1965. A method for estimating the potential fish production of north-temperate lakes. Transactions of the American Fisheries Society 94:214-218.

Tesch, F. W. 1971. Age and growth. In: Ricker, W. E. ed. Methods for assessment of fish production in fresh waters. Oxford, Blackwell Scientific Publications. 348p.

Thienemann, A. 1931. Der productionsbegriff in der biologie. Archives fur Hydrobiology 22:616-622.

WATERS, T. F. 1983. Replacement of brook trout by brown trout over 15 years in a Minnesota stream: production and abundance. Transactions of the American Fisheries Society 112:137-146.

Watson, D. J. \& BALON, E. K. 1984. Structure and production of fish communities in tropical rain forest stream of north Borneo. Canadian Journal of Fisheries and Aquatic Science 62:927-940.

Whitworth, W. E. \& Strange, R. J. 1983. Growth and production of sympatric brook and rainbow trout in a Appalachian stream. Transactions of the American Fisheries Society 112:469-475.

ZAR, J. H. 1999. Biostatistical analysis. New Jersey, PrenticeHall. $663 \mathrm{p}$.

ZipPIN, C. 1958. The removal method of population estimation. Journal of Wildlife Management 2:82-90. 